\title{
Estimation of plate material properties by means of a complex wavenum- ber fit using Hankel's functions and the image source method
}

\author{
N.B. Roozen \\ Laboratory of Acoustics, Division Soft Matter and Biophysics, Department of \\ Physics and Astronomy, KU Leuven, Celestijnenlaan 200D, 3001 Leuven, Bel- \\ gium \\ Q. Leclère \\ Univ Lyon, INSA-Lyon, Laboratoire Vibrations Acoustique, F-69621 Villeurbanne, \\ France \\ K. Ege \\ Univ Lyon, INSA-Lyon, Laboratoire Vibrations Acoustique, F-69621 Villeurbanne, \\ France \\ Y. Gerges \\ Univ Lyon, INSA-Lyon, Laboratoire Vibrations Acoustique, F-69621 Villeurbanne, \\ France
}

\begin{abstract}
This paper presents a new wave fitting approach to estimate the frequency dependent material properties of thin isotropic plate structures from an experimentally obtained vibrational field, exciting the plate at a single point. The method projects the measurement data on to an analytical image source model, in which Hankel's functions are used for a description of the wave fields emanating from the point of excitation, including the reflected wave fields from the edges of the finite plate. By minimizing the error between the projected field and the measured field, varying the complex wave number and the source strengths of the image sources, an optimum fit is searched for. Thus the source strengths of the image sources do not need to be determined theoretically, but are estimated from the fit on to the experimental data instead (thus avoiding difficulties in theoretically assessing the reflection coefficient of the edges of the plate). The approach uses a complex wavenumber fit, enabling the determination of the dynamic stiffness of the plate structure and its damping properties as function of frequency. The method is especially suited for plates with a sufficient amount of damping, excited at high frequencies.
\end{abstract}

Submitted to Journal of Sound and Vibration. Accepted 5 December 2016 http://dx.doi.org/10.1016/j.jsv.2016.11.037 


\section{Introduction}

In literature the wave number fitting approach is well-known. However, the combination of the Hankel's functions with the image source method to describe the (full or partial) wave field of a point excited plate, to pursue a wave fitting procedure on to this vibrational field with the aim to assess the isotropic material properties of the plate, is new. To put the proposed approach into perspective, below the relevant literature is discussed briefly.

Wave fitting approaches often consider plane damped waves of the form $\exp ( \pm i k x)$, sometimes referred to as inhomogeneous wave, where $k$ is the (complex valued) wavenumber, and $x$ is a spatial coordinate. For instance, McDaniel et al. [1] use these types of waves to estimate the dispersion relations of waves in damped 1D structures (i.e. a beam).

Berthaut et al. [2] consider plane waves of the form $\exp \{ \pm i k(\theta)(x \cos (\theta)+y \sin (\theta))\}$, to identify $\theta$-dependent dispersion equation of anisotropic panels. Here $x$ and $y$ are the plate coordinates, and $\theta$ is the angle of propagation. They demonstrate for a rib-stiffened panel that the wavenumber $k$ is indeed dependent upon propagation angle $\theta$ (considering the real part of the wavenumber only). Even though they also tested a steel panel with a bonded porous layer, damping factors were not presented in their paper, which was apparently more difficult.

Very recently, Cherif et al. [3] used plane waves of the same form, i.e. $\exp \{ \pm i k(\theta)(x \cos (\theta)+y \sin (\theta))\}$, to estimate the flexural wavenumber and the damping loss factor of plate structures in two dimensions from a displacement field measurement, which is essentially the same approach as followed by the previously described references. They applied the method to an isotropic aluminum panel and to orthotropic sandwich composite panels with a honeycomb core. Both flexural wavenumbers and damping loss factors were determined successfully.

Going back a bit further in time, Grosh and Williams [4] used the Prony method up to a frequency of $20 \mathrm{kHz}$ to estimate the dispersion relationships of a point driven cylindrical shell from the measured vibrational field. Basically, this also comes down to the use of waves of the form $\exp \left( \pm i k_{x} x \pm i k_{y} y\right)$.

The basic problem with planar waves of the type $\exp \left( \pm i k_{x} x \pm i k_{y} y\right)$ is that the actual vibrational field of a structure significantly deviates from a plane wave field near the point(s) of excitation. Some of the cited papers indeed mention that it is required to measure the steady state response at points not too close to the point(s) of excitation. Obviously, this is necessary because of the plane wave assumption, which is only valid in the far field. This requirement, however, is not necessary for the approach presented in this paper. The Green's function of a

Submitted to Journal of Sound and Vibration. Accepted 5 December 2016 http://dx.doi.org/10.1016/j.jsv.2016.11.037 
point excited plate that are used in this paper are also valid relatively close to the point of excitation, as it includes both propagating and evanescent waves.

The approach of Cuenca et al. [5] combines the use of the image source method and Hankel's functions to construct the Green's function of a finite plate with simply supported boundary conditions. Such a model is much better suited to represent the vibrational field of a point excited plate, especially in the higher frequency range, as the accuracy of the image source method is known to increase with frequency and damping [6]. Indeed, Cuenca and Simon [7] estimated the material properties of a point excited plate (with free boundary conditions) on the basis of a point mobility measurement, fitting the point mobility measurement data on to the image source method model.

The approach discussed in this paper is different from the approach used by Cuenca and Simon in that the (complete or partial) vibrational field of the plate is used in the fit procedure to extract the material properties. Green's functions are constructed on the basis of Hankel's functions (in two spatial dimensions), using the image source method.

Using the image source method to theoretically describe the wave field of a plate with a boundary condition which is different from a simply supported or roller boundary condition, is a difficult task (albeit not impossible, see [6, 8]). Moreover, in practice the reflection coefficients deviate from the theoretical prediction, because of non-modelled dissipation effects, introducing an uncertainty in the theoretical models. For this reason, in the present paper the source strengths of the image sources are not determined analytically, but are determined by fitting an image source model on the experimental data.

Contrarily to the classical spatial Fourier transform, the intrinsic advantage of the wave number domain fit procedure is a wavenumber resolution that is no longer limited by the length of the scan. This advantage of wave fitting procedures will be illustrated by means of experimental results in Section 4.2. Moreover, it is shown that the approach is capable of extracting both stiffness and damping properties of the plate, using only a limited number of image sources. The advantages of the Bayesian regularization is illustrated as well.

The paper is organized as follows. Section 2 deals with the theory of image sources for a point excited plate. Hankel's functions are used to approximate the Green's function of a finite plate with arbitrary boundary condition. In section 3 the validity of the image source method combined with the use of Hankel's functions is verified for a point excited rectangular plate with simply supported boundary conditions as well as for a plate with free edges. In section 4 the theory is applied to a sandwich plate, estimating both the real and imaginary parts of the

Submitted to Journal of Sound and Vibration. Accepted 5 December 2016 http://dx.doi.org/10.1016/j.jsv.2016.11.037 
wavenumber of the plate.

\section{Theory of the wave fitting methodology for a point excited finite plate}

The basic idea presented in this paper is to fit the measured vibrational field of a point excited finite plate, by means of an image source model, with the aim to identify the material properties of the plate. This theoretical section starts with a brief summary of the theory of Green's functions of an infinite plate and the construction of the Green's function of a finite plate by means of the image source method. After that, the projection of the measurement data onto an image source model of a finite plate is given.

\subsection{Green's functions of a point excited plate}

The equations of motion of an isotropic homogeneous plate of constant thickness, excited by a point force $\delta$ at $(x, y)=\left(x_{0}, y_{0}\right)$ in normal direction of the plate, is given by Kirchhoff's thin plate theory [9] as

$$
D\left(\nabla^{4}-k_{f}^{4}\right) w(x, y)=\delta\left(x-x_{0}, y-y_{0}\right)
$$

where $w(x, y)$ is the transverse motion of the plate, $k_{f}$ is the flexural wave number, defined by

$$
k_{f}=\left(\omega^{2} \frac{\rho h}{D}\right)^{1 / 4}
$$

where $\omega$ denotes the exciting angular frequency, $D$ is the dynamic flexural rigidity defined by

$$
D=\frac{E h^{3}}{12\left(1-\nu^{2}\right)}
$$

where $h$ is the plate thickness, $E$ is the Young's modulus and $\nu$ is the Poisson's ratio.

For a plate with infinite lateral dimensions, the solution of Eq. (1) is given by [5]

$$
G_{\infty}\left(x-x_{0}, y-y_{0}\right)=\frac{1}{8 k_{f}^{2} D}\left(H_{0}^{(1)}\left(k_{f} r\right)-H_{0}^{(1)}\left(i k_{f} r\right)\right)
$$




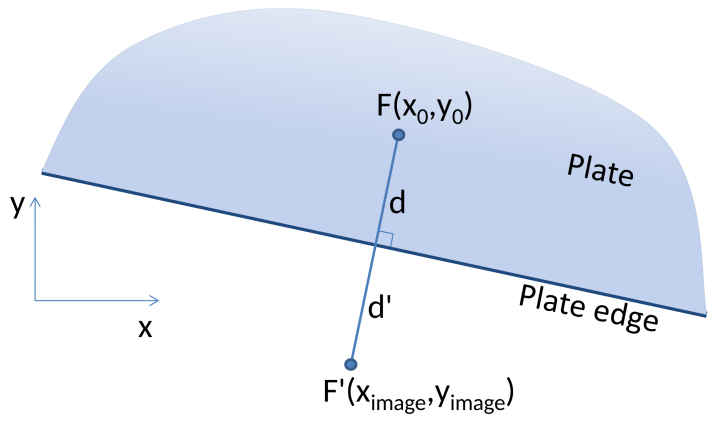

Figure 1: The image source method.

where $G_{\infty}$ is the Green's function of the infinite plate, $H_{0}^{(1)}$ is the cylindrical Hankel's function of the first kind of order 0 , and $r=\left\|\left(x-x_{0}, y-y_{0}\right)\right\|$ is the source-to-receiver distance.

For a finite plate, the Green's function can be approximated by means of the image source method [6, 10]. The image source method is basically a methodology to enforce the boundary conditions that should be satisfied at the edges of the finite plate. When dealing with a plate with straight edges, being excited by a point force $F$ at the coordinates $\left(x_{0}, y_{0}\right)$ (see Fig. 1), the image sources are fictitious point forces $F^{\prime}$ at the coordinates $\left(x_{\text {image }}, y_{\text {image }}\right)$, such that the distances $d$ and $d^{\prime}$ are equal. For point excitated plates the response of the individual image sources is given by the Green's function $G_{\infty}$, Eq. (4). This procedure is repeated for all edges of the finite plate. The response of the finite plate is equal to the sum of the individual contributions of the image sources. In case of opposing edges, this imaging procedure needs to be applied in a recursive manner. Depending on the magnitude of all image sources $F^{\prime}$, certain boundary conditions can be satisfied along the edge of the plate. In papers from Gunda the procedure is outlined for several types of boundary conditions [6, 8].

Submitted to Journal of Sound and Vibration. Accepted 5 December 2016 http://dx.doi.org/10.1016/j.jsv.2016.11.037 


\subsection{Projecting the measured vibrational field on an image source model of a finite plate}

Consider the measured out-of-plane displacement $w\left(x_{j}, y_{j}, \omega\right)$ of an harmonically excited, finite plate, at discrete measurement positions $\left(x_{j}, y_{j}\right), j=1 \ldots M$, on the plate surface. The vibrational field of the plate in the measurement area (being a partial area or the complete area of the plate) is approximated by means of a linear combination of image sources:

$$
\tilde{w}\left(x_{j}, y_{j}, \omega\right)=\sum_{n=1}^{N} \alpha_{n}(\omega) \phi_{n}\left(x_{j}, y_{j}\right)
$$

where $\alpha_{n}$ is the strength of each image source and $\phi_{n}$ the Green's function of an infinite plate:

$$
\phi_{n}\left(x_{j}, y_{j}\right)=G_{\infty}\left(x_{j}-x_{0}(n), y_{j}-y_{0}(n)\right)
$$

where $x_{0}(n)$ and $y_{0}(n)$ are the $x$ and $y$-locations of the $n$-th image source. This Green's function includes both propagating and evanescent waves. Considering all $M$ measurement positions on the plate and $N$ image sources, Eq. (5) can be written in matrix notation as

$$
\tilde{\mathbf{w}}=\boldsymbol{\Phi} \alpha
$$

where $\tilde{\mathbf{w}}$ is a vector containing the projected displacements $\tilde{w}\left(x_{j}, y_{j}, \omega\right), \boldsymbol{\Phi}$ is a matrix containing the vectors of the image source Green's functions, $\phi_{n}$, and $\boldsymbol{\alpha}$ is a vector containing the contribution strengths $\alpha_{n}(\omega)$. For brevity, the dependence of the variables on $\omega$ and (measurement) position will be dropped. For the practical implementation of the method it should be mentioned that the factor $1 /\left(8 k_{f}^{2} D\right)$ in $G_{\infty}$ (Eq. (4)) can safely be ignored to construct matrix $\Phi$.

As the number of measurement points (i.e. the length of vector w) considered in this work is much larger than the number of unknown contribution strengths (i.e. the length of vector $\boldsymbol{\alpha}$ ), the vector of contribution strengths $\boldsymbol{\alpha}$ needs to be determined by means of a generalized inverse approach:

$$
\boldsymbol{\alpha}=\boldsymbol{\Phi}^{+} \mathbf{W}=\left(\boldsymbol{\Phi}^{*} \boldsymbol{\Phi}\right)^{-1} \boldsymbol{\Phi}^{*} \mathbf{W}
$$

where $\Phi^{+}$is the pseudo inverse of matrix $\boldsymbol{\Phi}$. This approach finds the solution in the least squares sense, i.e. the solution of the following minimization problem

$$
\boldsymbol{\alpha}=\operatorname{argmin}\left(\|\mathbf{w}-\boldsymbol{\Phi} \boldsymbol{\alpha}\|^{2}\right)
$$

Submitted to Journal of Sound and Vibration. Accepted 5 December 2016 http://dx.doi.org/10.1016/j.jsv.2016.11.037 
However, in some situations, the condition number of matrix $\Phi$ can be relatively high, inducing a strong sensitivity of the approach. This is typically the case when increasing the number of image sources to be quantified. For instance, if the waves originating from two image sources have almost equal incidence angles upon the measurement area, their contributions to the vibration field will be too similar to be distinguished. In such a situation, the condition number can increase dramatically, leading to strong (non physical) overestimations of the recovered source strengths. A classical solution to overcome this issue is to use regularisation. The Tikhonov regularisation adds a term to the minimization problem (9), proportional to the solution's norm:

$$
\boldsymbol{\alpha}=\operatorname{argmin}\left(\|\mathbf{w}-\boldsymbol{\Phi} \boldsymbol{\alpha}\|^{2}+\lambda^{2}\|\boldsymbol{\alpha}\|^{2}\right),
$$

giving a solution that is obtained using the regularized pseudo-inverse:

$$
\boldsymbol{\alpha}=\boldsymbol{\Phi}^{+\lambda} \mathbf{w}=\left(\boldsymbol{\Phi}^{*} \boldsymbol{\Phi}+\lambda^{2} \mathbf{I}\right)^{-1} \boldsymbol{\Phi}^{*} \mathbf{w} .
$$

The choice of the regularization parameter $\lambda$ results from a trade-off between the fidelity to measurements and the minimisation of the source strengths. Its determination can be processed using various approaches, one can cite L-curve or generalized cross-validation (GCV) methods that have been successfully implemented in dynamic load identification problems [11]. In this work, a criterion based on a Bayesian formalism of the inverse problem is used. This approach has been developed for acoustic imaging applications [12], and has been found to behave more robustly than other approaches [13]. The Bayesian formalism, as well as the resulting cost function that has been used in this work are briefly described in appendix A.

Finally, the projected vibrational field $\tilde{\mathbf{w}}$ can be computed using

$$
\tilde{\mathbf{w}}=\boldsymbol{\Phi} \Phi^{+\lambda} \mathbf{w}
$$

The adequacy between matrix $\Phi$ and measurements $w$ is quantified by the following normalized reconstruction error

$$
e=\frac{\|\mathbf{w}-\tilde{\mathbf{w}}\|^{2}}{\|\mathbf{w}\|^{2}}
$$

where $\|\ldots\|$ denotes the Euclidean norm of a matrix. Note that $\mathbf{w}$ and $\tilde{\mathbf{w}}$ are a function of both frequency and space. The error $e$ is only a function of frequency.

The solution of Eq. (11) gives the (complex valued) source strengths of the image sources and the main source, that fits the measurement data best (with the

Submitted to Journal of Sound and Vibration. Accepted 5 December 2016 http://dx.doi.org/10.1016/j.jsv.2016.11.037 
regularization constraint that enforces the amplitudes of the source strengths not to be too high). The error $e$ also depends upon the (complex valued) flexural wave number $k_{f}$ used to build the 'test wave' fitting function matrix $\Phi$. The numerical procedure encompasses the minimization of the reconstruction error $e$, selecting an optimal value for $k_{f}$ from a large set of candidate values for $k_{f}$ sampled in the complex plane.

Using this approach, a single wavenumber $k_{f}$ is found for each exciting angular frequency $\omega$, as it is assumed that a single flexural wave is propagating. This is at one hand a limitation of the approach, on the other hand it allows a very accurate estimation of the wavenumber $k_{f}$, provided the assumption of a single flexural wave is valid for the studied case. The wavenumber domain resolution $d k$ thus obtained is much better by far, as compared to the wavenumber domain resolution that can be obtained with the spatial Fourier method [14], which is limited by the fundamental relationship $d k_{x, y} \propto 2 \pi / L_{x, y}$, where $L_{x}$ and $L_{y}$ are the dimensions of the measurement area in $x$ - and $y$-direction, respectively, as will be shown in Section 4.2 .

Once the optimum values for the image source strengths and the wavenumber $k_{f}$ are found, the dynamic flexural rigidity $D$ of the plate can be determined as function of frequency (cfr. Eq. (2)):

$$
D=\frac{\omega^{2} \rho h}{k_{f}^{4}}
$$

The structural damping is included in the dynamic flexural rigidity $D$ by writing (using the $\exp (-i \omega t)$ convention):

$$
D=D_{0}(1-i \eta)
$$

where $\eta$ is the structural loss factor. From Eq. (14) and (15) it can be derived that the loss factor $\eta$ is related to the complex wavenumber $k_{f}$ as follows [15]:

$$
\eta=\frac{\Im\left(k_{f}^{4}\right)}{\Re\left(k_{f}^{4}\right)}
$$

\section{Validation of the image source method}

The approach presented in this paper relies on an image source model, using Hankel's functions as a basis of the image source responses, describing the vibrational

Submitted to Journal of Sound and Vibration. Accepted 5 December 2016

http://dx.doi.org/10.1016/j.jsv.2016.11.037 


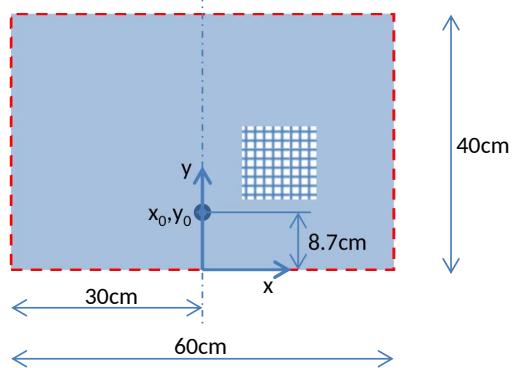

a)

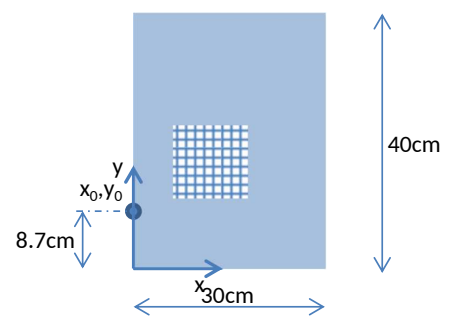

b)

Figure 2: Rectangular plate. Excitation point in normal direction at $x_{0}=0, y_{0}=8.7$ $\mathrm{cm}$, as indicated. Vibrational data obtained on a mesh as indicated, from $x=6.17$ $\mathrm{cm}$ to $17.86 \mathrm{~cm}$, in 41 steps, from $y=11.08 \mathrm{~cm}$ to $22.19 \mathrm{~cm}$, in 39 steps. Total number of points (i.e. the length of vector w): 1599. a) Simply supported plate of dimension $60 \times 40 \times 0.15 \mathrm{~cm}$ (dashed line indicates simply supported boundary condition); b) plate with free edges of dimension $30 \times 40 \times 0.15 \mathrm{~cm}$.

field of a point excited finite plate. In this section the validity of the image source method combined with the use of Hankel's functions is discussed.

The magnitude of the image sources are particularly simple for a point excited rectangular plate with simply supported boundary conditions. In this case the sources should be the same in magnitude (and changing 180 degrees in phase each time when passing an edge of reflection), as can be proven by theory [10]. The fitting procedure outlined in Section 2 will first be applied to this case, as a validation of the approach. However, as in practice a free boundary condition is much more easy to realize, a plate with free edges will be discussed as well.

For the simply-supported rectangular plate an analytical model was used to generate the vibrational field (employing Green's kernel of a simply supported rectangular damped plate [16]). The configuration is shown in Fig 2a. The vibrational field due to a point force excitation at $(x, y)=\left(x_{0}, y_{0}\right)$ was calculated analytically on a discrete mesh as shown in this figure as well. Using this discrete set of (complex valued) vibrational data, the fitting procedure of Section 2 was used to determine the strengths of the image sources. An image source model with 9 sources was employed for this purpose (ID's 1-9 in Fig 3 a).

The vibrational field that computed analytically, w, as well as the projected vibrational field, $\tilde{\mathbf{w}}$ (see Eq. (7)), are shown in Figure 4 for a frequency of $15 \mathrm{kHz}$. This figure shows that the typical variation of the amplitude of the vibration due

Submitted to Journal of Sound and Vibration. Accepted 5 December 2016 http://dx.doi.org/10.1016/j.jsv.2016.11.037 


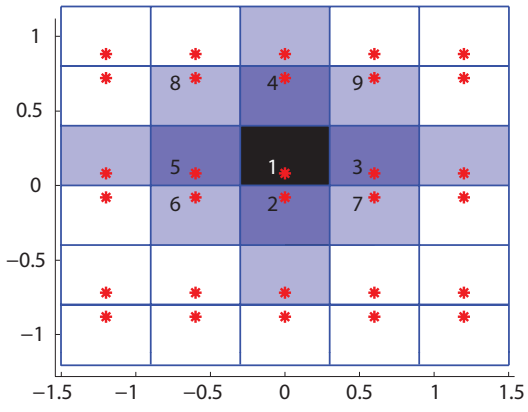

a)

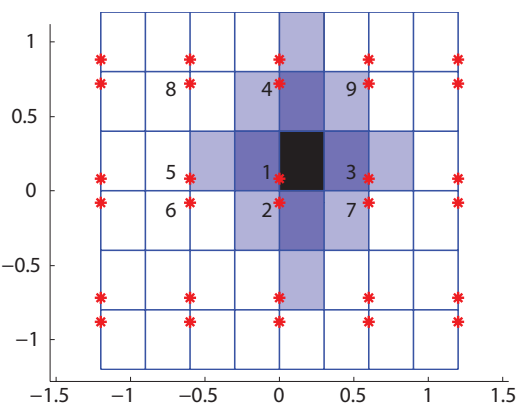

b)

Figure 3: (color online) The image source model. a) Point excited simply supported plate (cfr. Fig 2a). b) Point excited free plate (cfr. Fig 2b). The red dots indicate the position of the (image) sources. The black area indicates the physical plate (its edges giving rise to first reflections). The dark blue areas indicate the areas from which the second reflection occurs. The light blue areas indicate the areas from which the third reflection occurs.

to constructive and destructive interference of the direct waves and the reflected waves is very well reproduced by the fitted image source model with 9 sources. Regularization was not required in this case as the analytical 'measurement' data was exact. The error $e(\mathrm{Eq} .(13))$ is as low as 0.0024 .

As can be seen from Figure 5, all the source strengths have about the same magnitude, as it should be, whilst the phase of the sources are in-phase or out-ofphase with each other. This is in line with theory, thus validating the approach to determine the source strengths from the vibrational field of a simply supported plate, employing an image source model on the basis of Hankel's functions. The amplitudes of the sources are inversely proportional with frequency, as the analytical model assumes a constant force. This is also in agreement with theory.

For a rectangular plate with free edges, analytical solutions are difficult to find. For practical reasons we resorted to the finite element method (FEM). The geometry of the plate and the point of excitation are indicated in Fig. 2b. A structural loss factor $\eta$ of 5\% was specified in the FEM model, which is of the same order of magnitude as the structural loss factor of the sandwich plate that was examined experimentally (to be discussed in Section 4). As the frequencies of interest are rather high, a FEM model was employed with a large amount of elements (56040 quadrilateral shell elements) and a significant number of degrees of freedom (339126). The vibrational field $w$ as calculated by the FEM model is

Submitted to Journal of Sound and Vibration. Accepted 5 December 2016 http://dx.doi.org/10.1016/j.jsv.2016.11.037 


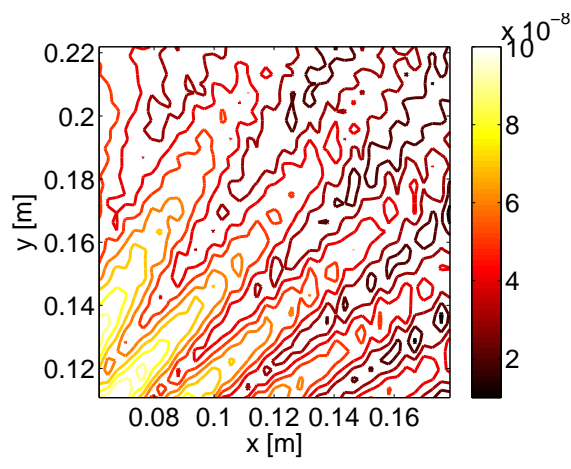

a)

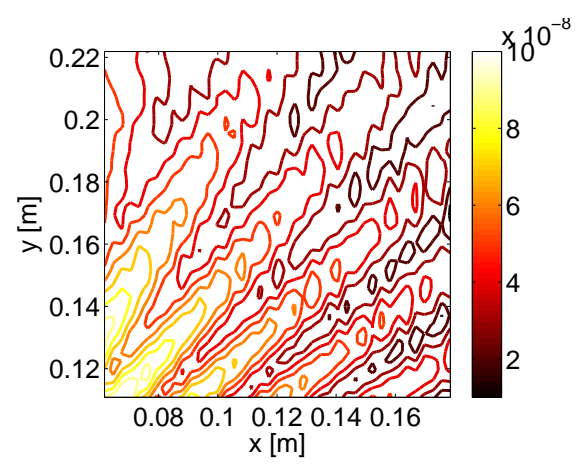

b)

Figure 4: (color online) Contour lines of absolute vibration levels of a simply supported plate (Fig 2a), in normal direction, at $15 \mathrm{kHz}$. a) Analytical data; b) Projection of the analytical data on an image source model with 9 sources, without regularization (error $e=0.0024$ ).

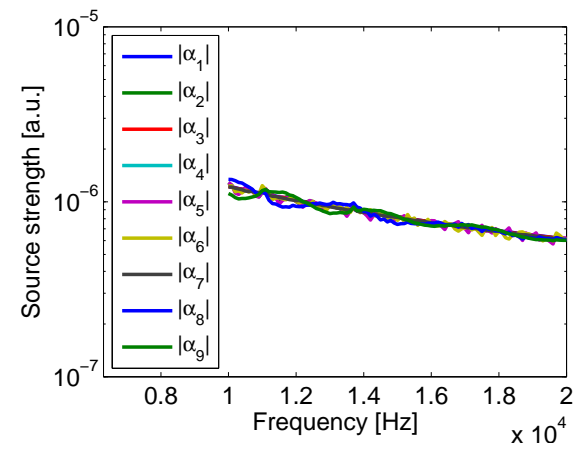

a)

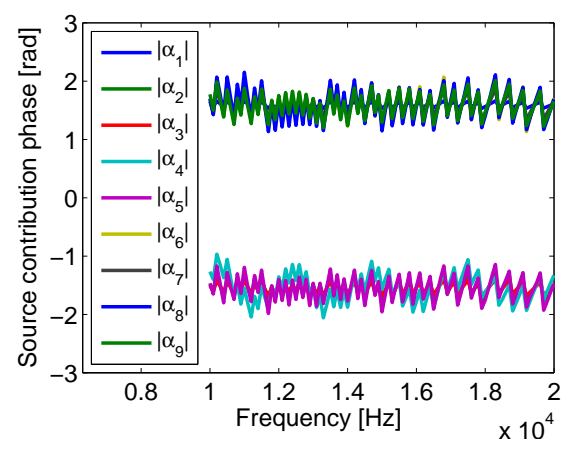

b)

Figure 5: (color online) Source contributions $\alpha$ for the reconstruction of the analytically generated data of a simply supported plate (Fig 2 a), using an image source model with 9 sources. a) Absolute value [a.u.]; b) Phase [rad].

shown in Fig. 6e for a frequency of $15 \mathrm{kHz}$.

The projected field, $\tilde{\mathbf{w}}$ (see Eq. (7)), for a frequency of $15 \mathrm{kHz}$, is shown in Fig. 6a-d for a varying number of image sources, illustrating the convergence behavior as function of the number of the Hankel's function image sources. For the case of an image source model with 9 sources, reference is made to Fig 3 b, which shows the location of the 9 sources. The typical interference pattern in the magnitude of the vibrational field can very well be described with the image

Submitted to Journal of Sound and Vibration. Accepted 5 December 2016 http://dx.doi.org/10.1016/j.jsv.2016.11.037 


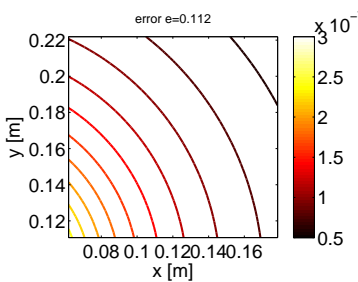

a)

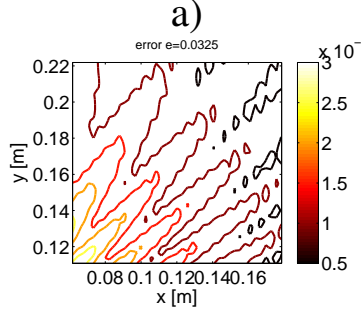

c)

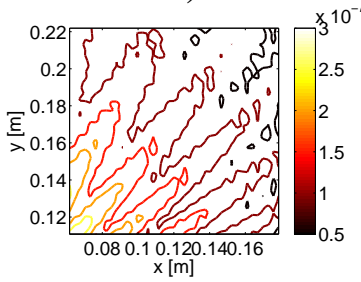

e)

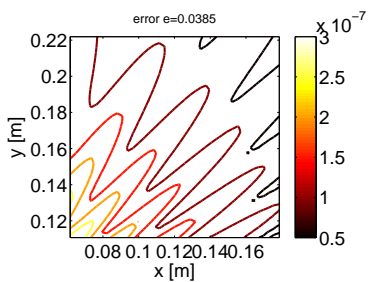

b)

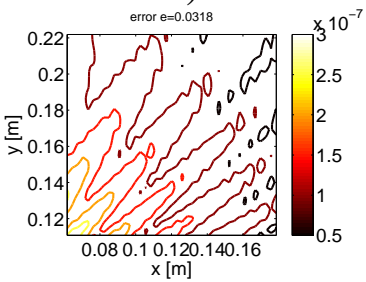

d)

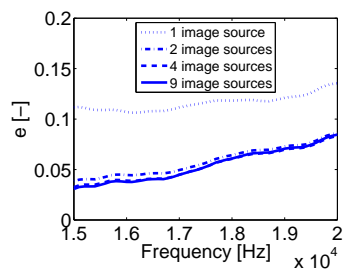

f)

Figure 6: (color online) Contour lines of absolute vibration levels (a.u.) of a plate with free edges, in normal direction, at $15 \mathrm{kHz}$. Projection of the numerically obtained data on an image source model, using Bayesian regularization, with a) 1 source (ID 1, error $e=0.11$ ); b) 2 sources (ID's 1-2, error $e=0.039$ ); c) 4 sources (ID's $1-4$, error $e=0.033$ ); d) 9 sources (ID's 1-9, error $e=0.032$ ). e) Numerically obtained FEM data. f) Error $e$ as function of frequency, as defined by Eq. (13).

source method for this free boundary case as well. The convergence behavior in terms of the reconstruction error $e$ (Eq. (13) $)$ as function of frequency is shown in Fig. 6f.

The error $e$ (Eq. (13) ) appears to be higher as compared to the error of the simply supported plate when using an analytical model. To investigate this in more detail, the FEM simulations were also performed for the simply supported plate, and the result were fitted on the image source Hankel model. Whilst for the analytical solution the error was 0.0024 , it was found that the fitting error for the FEM data was about 0.03 , using the same number of image sources. So, it can be concluded that the increase in error from approximately 0.003 to approximately

Submitted to Journal of Sound and Vibration. Accepted 5 December 2016

$$
\text { http://dx.doi.org/10.1016/j.jsv.2016.11.037 }
$$


0.03 is indeed due to the noise on the numerically generated data.

It can be concluded that nine sources are sufficient to describe the vibrational field of a point excited plate with the given geometry and boundary conditions, in the frequency range of interest. Analytically generated data gives a lower projection error $e$ as compared to data generated by a FEM model.

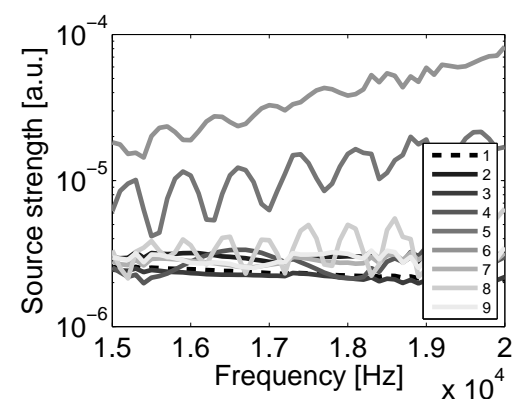

a)

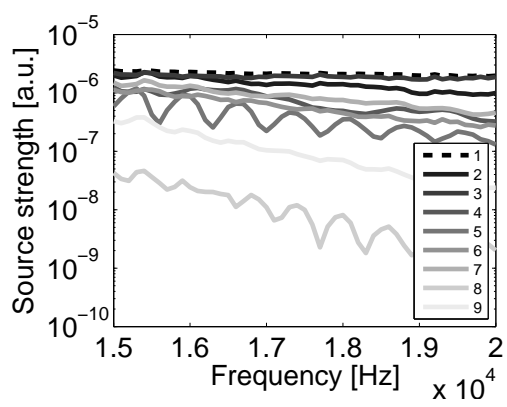

b)

Figure 7: (color online) Image source strengths $|\alpha|$ of the 9 image sources model, as function of frequency, using the numerically obtained vibration patterns of a plate with free edges. a) Without regularization; b) with Bayesian regularization. Note that the y-axes are different.

Due to the numerical inaccuracies that are introduced by the FEM-model, regularization was required. To illustrate this, Fig. 7 shows the source strengths of the image sources for the image source model with nine image sources, with and without regularization. Without regularization, problems especially occur for the image sources that are relatively far away from the point of excitation. For instance, from Fig. $7 \mathrm{~A}$ it can be seen that, without regularization, the image source strengths of the image sources further away from the main source (high ID number) are much larger than the strength of the main source (ID 1). This is caused by the fact that their contributions to the vibrations in the measurement area are very similar to each other (plane waves), causing a bad conditioning of the matrix $\Phi$ to be inverted. When using Bayesian regularization, this non-physical behavior can be suppressed effectively, resulting in a reduction of the image source strength with distance from the main source (see Fig. 7p).

The purpose of this paper is to estimate the material properties of the plate, from the projection of the measurement data on a numerical (image source) model. Basically the optimum value of the complex wavenumber $k$, from which the material properties can be determined, is searched for to minimize the projection error

Submitted to Journal of Sound and Vibration. Accepted 5 December 2016 http://dx.doi.org/10.1016/j.jsv.2016.11.037 


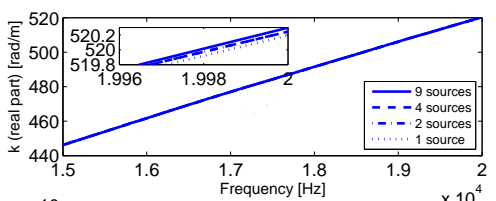

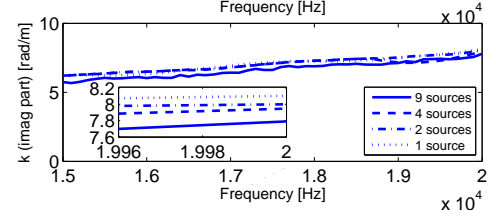

a)
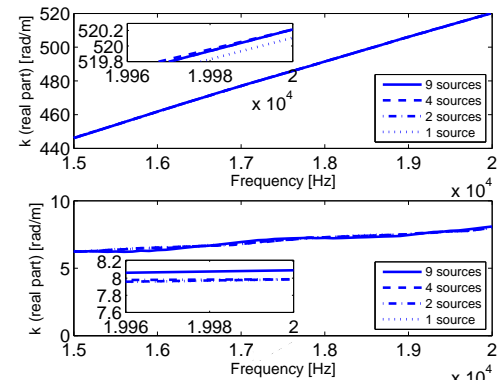

b)

Figure 8: The influence of the number of image sources on the estimate of the real (top) and imaginary (bottom) wavenumber for a point excited plate with free edges. a) with Bayesian regularization; b) Without regularization.

$e$. The influence of the number of image sources on the estimate of the complex wavenumber is shown in Fig. 8 a for the same point excited plate, with Bayesian regularization. For the real part and the imaginary part of the wavenumber the maximum variation of the estimate is approximately less than $0.2 \mathrm{rad} / \mathrm{m}$ and 0.7 $\mathrm{rad} / \mathrm{m}$, respectively. For the real part of the wavenumber this is relatively speaking very small (less than $0.5 \%$ ), whilst for the imaginary part it is more significant (less than 10\%). Thus, giving the relationships between the wavenumber estimate and the material properties, as given in Eqs. (15) and (16), the influence on the estimate of the Young's modulus and the loss factor, will be less than $4 \times 0.5 \%=$ $2 \%$ and $10 \%$, respectively. It shows that the number of images used in the image source model does not have a profound impact upon the estimate of the Young's modulus. The influence on the estimate of the loss factor, is more significant, but less than $10 \%$.

Figure $8 \mathrm{~b}$ shows the influence of the number of image sources on the estimate of the complex wavenumber, when not using regularization. The errors are approximately of the same order of magnitude as compared to Fig. 89, albeit that the convergence is slower. For instance the imaginary part of the wavenumber stays relatively high for the case without regularization, whilst with Bayesian regularization the imaginary part converges to a lower value when increasing the number of image sources. A lower value of the imaginary part of the wavenumber is believed to be physically more correct.

In summary it can be concluded that regularization does not have a significant effect on the estimate of the flexural wavenumbers, which makes the method

Submitted to Journal of Sound and Vibration. Accepted 5 December 2016 http://dx.doi.org/10.1016/j.jsv.2016.11.037 
not very sensitive to the regularization approach taken. This does not, however, alter the fact that the use of proper regularization is highly preferable in order to allow for a proper description of the physical behavior of the system. Although in our specific application the regularization did not have a strong effect, it could be the case in other situations, with different plate geometry, boundary conditions, etc.

\section{Experiments}

\subsection{Test set-up}

To illustrate how the methodology proposed in this paper can be easily applied to common engineering problems which may arise in the vibroacoustic community, we made the choice to conduct measurements on a typical three layer sandwich plate used in vehicle industry. The plate under consideration consists of a thin steel layer of $0.18 \mathrm{~mm}$ thickness, a polymer layer of $0.69 \mathrm{~mm}$ thickness and a thin steel layer of $0.18 \mathrm{~mm}$ thickness. The overall dimensions of the plate are $30 \mathrm{x} 40 \mathrm{~cm}^{2}$. The plate was hanging on two thin nylon wires (for which two small holes were drilled in the plate), thus realizing a practically free boundary condition. The plate was excited at its edge by means of a Bruel\&Kjaer mini-shaker type 4810 . The shaker was driven by a white noise signal (5-20 kHz). The structural vibrations of the plate were measured by means of a scanning laser Doppler vibrometer, consisting of a Polytec PSV-400 scanning head and a Polytec OFV-5000 controller. Only a part of the plate was measured by means of the scanning laser Doppler vibrometer, as indicated in Fig. 2b and in Fig 9] (scanning area approximately 11.7 $\mathrm{x} 11.1 \mathrm{~cm}^{2}$ ). The measurements were taken sequentially in time, using the signal that was sent to the shaker as a reference signal, allowing the measurement of both the amplitude and phase of the vibrational response of the plate. Alternatively, a force cell could have been used for this purpose. However, it is not a necessity to do so, as our interest was the measurement of the vibrational field of the plate to extract wavenumber properties from it. This does not require the excitation force to be known.

The image source model is schematically depicted in Fig. 3p, showing the main excitation source (ID number 1) and a number of image sources. An image source model with 9 sources is used in this section, as it was concluded in Section 3 that for this type of excitation and plate geometry with free edges, 9 images sources are sufficient.

Submitted to Journal of Sound and Vibration. Accepted 5 December 2016 http://dx.doi.org/10.1016/j.jsv.2016.11.037 


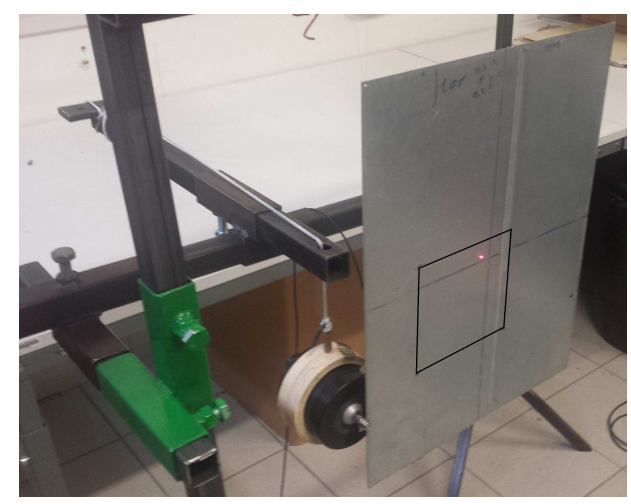

Figure 9: (color online) Measurement test set-up. Plate hanging on thin wires, excited by mini-shaker. The black line on the plate indicates the measurement area.

\subsection{Results}

The measured vibrational field is shown in Fig. 10a for one frequency only (15 $\mathrm{kHz}$ ), as an example. Varying the real and imaginary parts of the wavenumber of the fitting functions contained in $\Phi$, the optimum value that was found using an image source model is $k=\left(436.3+6.1\right.$ i) $\mathrm{m}^{-1}$ for this frequency. The projected vibrational field $\tilde{\mathbf{w}}\left(x_{i}, y_{i}\right)$ at $15 \mathrm{kHz}$ is presented in Fig. 10p. The typical ampli-

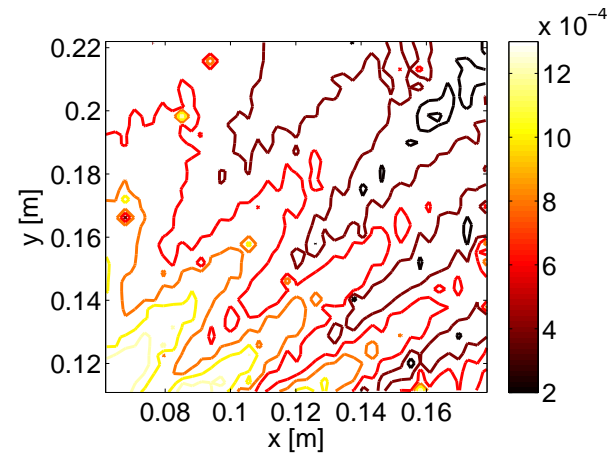

a)

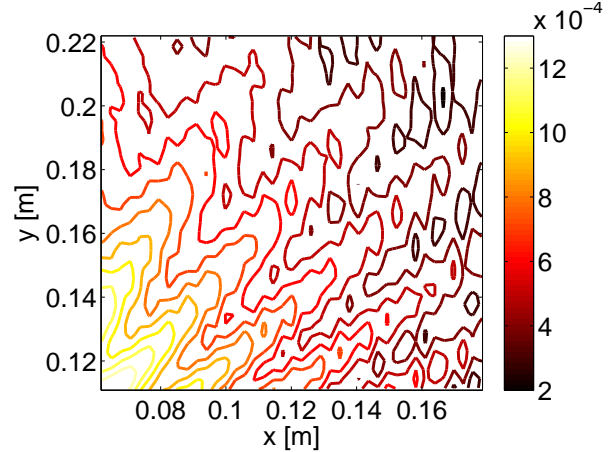

b)

Figure 10: (color online) Contour lines and surface plots of experimentally obtained absolute vibration levels of a plate with free edges (Fig 2b), in normal direction, at $15 \mathrm{kHz}$. a) Experimental data; b) Projection of the data using an image source model with 9 sources (ID 1-9, error $e=0.11$ ) using Bayesian regularization.

Submitted to Journal of Sound and Vibration. Accepted 5 December 2016 http://dx.doi.org/10.1016/j.jsv.2016.11.037 
tude variation due to interference effects of the direct vibrational field of the main source and the vibrational field resulting from the plate edge reflections (which are described by the image sources), are reasonably represented by the image source approach, as expected from the results that were shown in Section 3 .

Using Bayesian regularization, the optimum value of the complex wavenumber $k$ of the fitting functions contained in $\Phi$ is searched for, minimizing the error $e$ of Eq. (13). The error $e$ as function of frequency and as function of the real part of the test wavenumber $k$ (for the optimum value of the imaginary part of $k$ ), is shown in Fig. 11月. The error $e$ as function of frequency and as function of the imaginary part of the test wavenumber $k$ (for the optimum value of the real part of

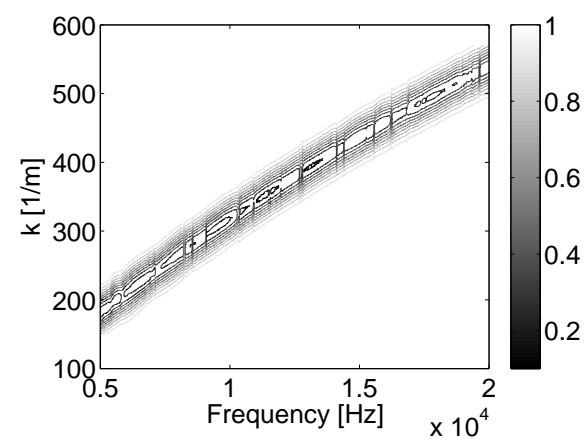

a)

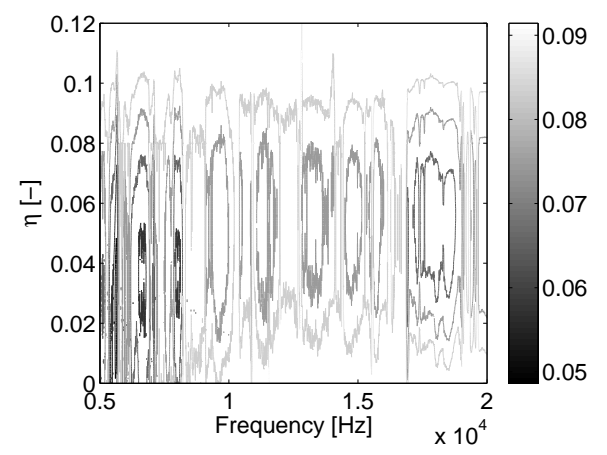

c)

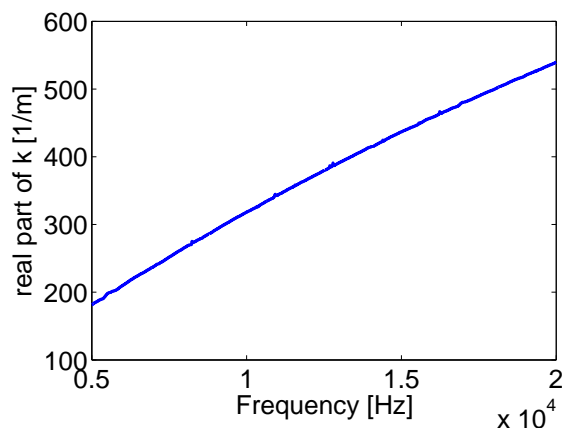

b)

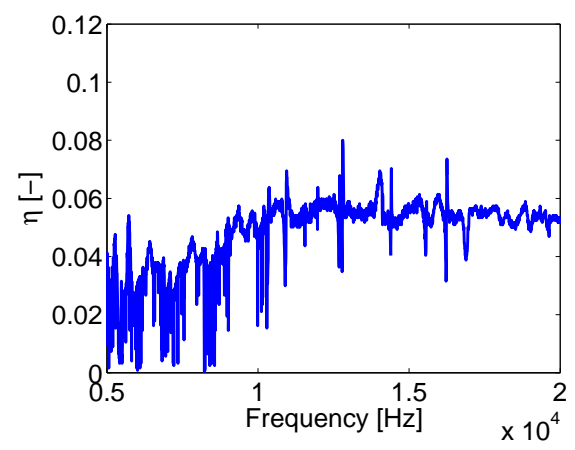

d)

Figure 11: (color online) Fitting error $e$ (defined in Eq. $(13)$ ) as function of frequency and as function of a) the real part of $k$ (for the optimum value of the imaginary part of $k$ ), and c) as function of the loss factor $\eta$ (for the optimum value of the real part of $k$ ). Resulting optima of b) the real part of the wavenumber $k$, and d) the loss factor $\eta$.

Submitted to Journal of Sound and Vibration. Accepted 5 December 2016 http://dx.doi.org/10.1016/j.jsv.2016.11.037 


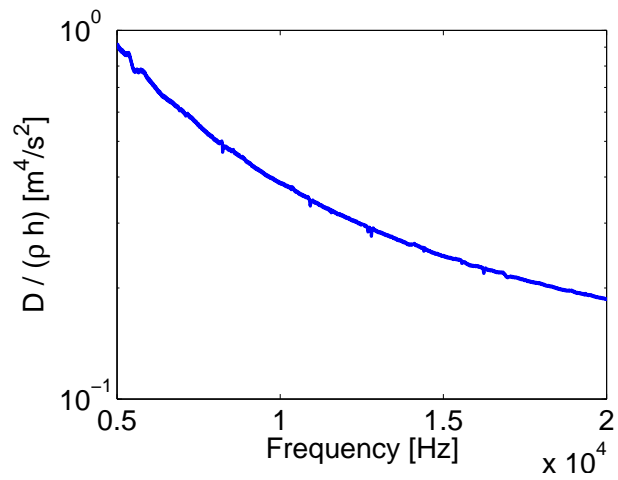

a)

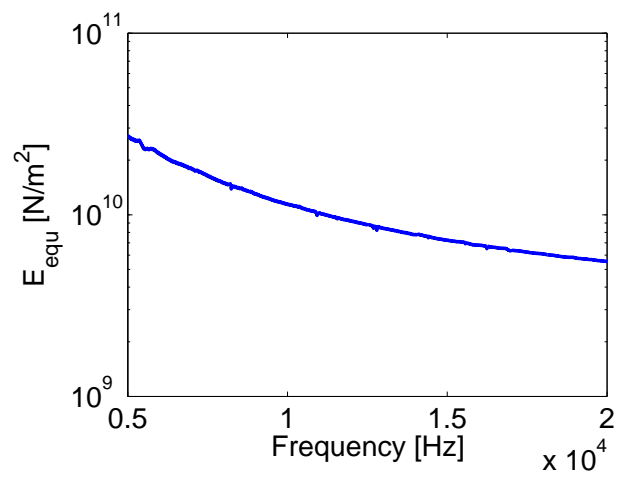

b)

Figure 12: Plate stiffness estimations. a) Dynamic flexural rigidity $D$ divided by $\rho h ; \mathrm{b})$ Equivalent Young's modulus $E$.

$k$ ), is shown in Fig. 11k. The corresponding optima of the real and imaginary parts of the wavenumber $k$ are shown in Fig. $11 \mathrm{~b}$ and Fig. $11 \mathrm{~d}$, respectively. Here, the structural loss factor $\eta$ is shown, instead of the imaginary part of the wavenumber $k$, exploiting relationship given by Eq. (16).

Having an estimate of the wavenumber $k$ as function of frequency, the dynamic flexural rigidity $D$ can be computed using Eq. (14). When applied to composite panels, the Kirchhoff's thin plate theory (Eq. (1)) can still be used if the vibrational behavior of the plate is assumed to be governed by flexural waves. However, the structure being composed of several layers of materials with different moduli, the relationship between the rigidity $D$ and Young's modulus $E$ is not valid anymore. A Young's modulus can still be estimated using Eq. (3), but it correspond to the Young's modulus of an equivalent homogeneous plate of same thickness and mass per unit area, that would exhibit the same dynamic flexural rigidity at one given frequency. This Young's modulus, that we call in this study the "equivalent Young's modulus", is not expected to be independent of the frequency anymore, as it was the case for homogeneous plates.

The estimate of the dynamic flexural rigidity $D$ is shown in Fig. 12a. Assuming a density $\rho$ of $3055 \mathrm{~kg} / \mathrm{m}^{3}$ and a thickness of $1.05 \mathrm{~mm}$ (measured values), the equivalent Young's modulus of the three-layer plate is computed by means of Eq. (3), shown in Fig. 12 p.

The confidence in the least squares fit results can be estimated as follows. Let's assume that each data point, as measured by the laser Doppler vibrometer,

Submitted to Journal of Sound and Vibration. Accepted 5 December 2016 http://dx.doi.org/10.1016/j.jsv.2016.11.037 
is drawn from a Gaussian distribution. Defining a (reduced, i.e. corrected with a factor $1 /(M-P))$ goodness-of-fit parameter $\chi^{2}$ :

$$
\chi^{2}=\frac{1}{M-P} \Sigma_{i, j}\left\{\frac{\left[w\left(x_{i}, y_{j}\right)-\tilde{w}\left(x_{i}, y_{j}\right)\right]^{2}}{\sigma_{i j}^{2}}\right\}
$$

where $w\left(x_{i}, y_{j}\right)$ is the measured data at measurement point $\left(x_{i}, y_{j}\right)$, having a standard deviation $\sigma_{i j}, \tilde{w}\left(x_{i}, y_{j}\right)$ is the fitted data at that point, $M$ is the number of measurement data points and $P$ is the number of fit parameters. Since the $\chi^{2}$ function is minimum at the best fitting parameter values, it can be approximated to second order as [17]

$$
\chi^{2}=\frac{\left(a_{k}-a_{k}^{o p t}\right)^{2}}{(M-P) \sigma_{k}^{2}}+C
$$

where $a_{k}$ is the $k$-th fitting variable, $a_{k}^{o p t}$ is the optimum value of the $k$-th fitting variable that minimizes $\chi^{2}, \sigma_{k}$ is the standard deviation of the $k$-th fitting variable, and $C$ is some constant, depending upon the other fitting variables. In case of
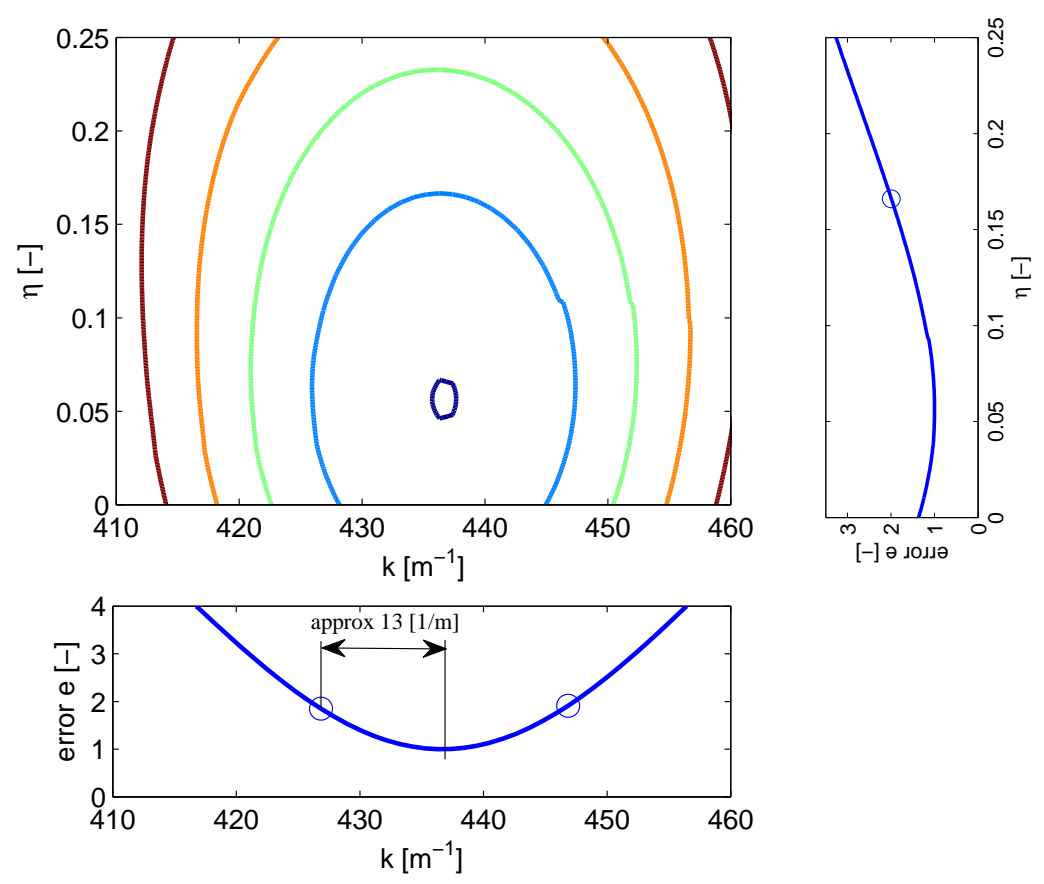

Figure 13: (color online) Error $e=\left(w_{i j}-\tilde{w}\left(x_{i}, y_{j}\right)\right) / w_{i j}$ as function of fitting parameter $k$ (real part) and as function of $\eta$, for a frequency of $15 \mathrm{kHz}$.

Submitted to Journal of Sound and Vibration. Accepted 5 December 2016 http://dx.doi.org/10.1016/j.jsv.2016.11.037 


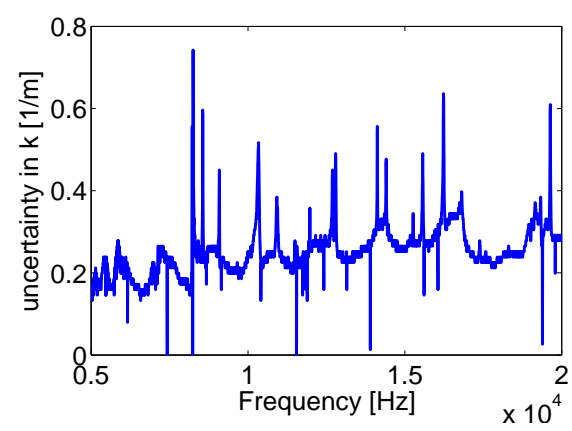

a)

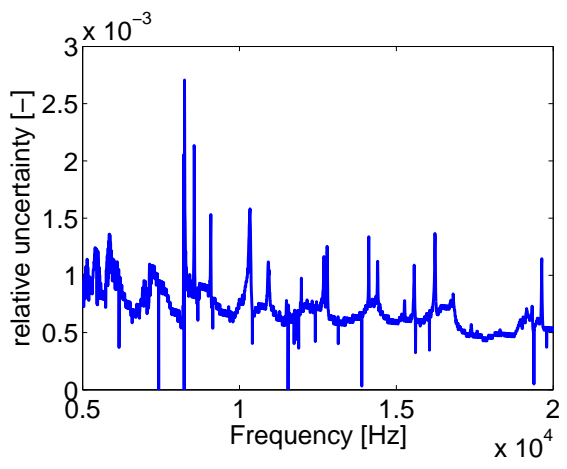

b)

Figure 14: Uncertainty estimate in $k$ (real part). a) absolute uncertainty. b) relative uncertainty.

no systematic errors, it can be expected that the deviations $\left(w\left(x_{i}, y_{j}\right)-\tilde{w}\left(x_{i}, y_{j}\right)\right)$ for the best fitting parameters are of the order of the expected standard deviations $\sigma_{i j}$, so that $C$, the minimum value of $\chi^{2}$, is of the order of unity. In this study it is assumed that the minimum value of $\chi^{2}$ equals 1 . From statistical theory it is know that an increase of 1 standard deviation $\left(\sigma_{k}\right)$ in the parameter from the value $a_{k}^{o p t}$ at the minimum increases $\chi^{2}$ by $1 /(M-P)$ [17]. Thus, the uncertainty in the fitting parameter $k, \sigma_{k}$, can be determined by plotting the error function $e=\left\{w\left(x_{i}, y_{j}\right)-\tilde{w}\left(x_{i}, y_{j}\right)\right\} / w\left(x_{i}, y_{j}\right)$ as function of this fitting parameter, and searching for the values of $a_{k}$ for which $e$ is increased by a factor 2 relative to the minimum of $e$. The standard standard deviation $\left(\sigma_{k}\right)$ can be obtained by dividing this range by $\sqrt{M-P}$.

Figure 13 shows the error $e$ (defined in Eq. (13) as function of the fitting parameters $k$ (real part) and $\eta$, for only one frequency: $15 \mathrm{kHz}$. Following the above described procedure, it can be seen that at this frequency the least squares uncertainty in the wavenumber estimate is about $\pm 13 / \sqrt{(M-P)} \approx 0.3 \mathrm{~m}^{-1}$, with $M=39 \times 41=1599$ (the number of measurement points of the LDV-grid) and $P=2+18$ ( 2 fitting parameters for the complex valued wavenumber $k$ and the real and imaginary part of the source and the 9 image sources). Evaluating the uncertainty for all frequencies gives the result as shown in Fig. 14.

When only one parameter is varied, and the others are kept at the best fitting value, a least squares parabola is retrieved for each fitting variable, as shown in Fig. 13. From the shape of the contour lines, being an oval with principal axes along the two fitting parameters $k$ and $\eta$, it can be concluded that there is not a

Submitted to Journal of Sound and Vibration. Accepted 5 December 2016 http://dx.doi.org/10.1016/j.jsv.2016.11.037 


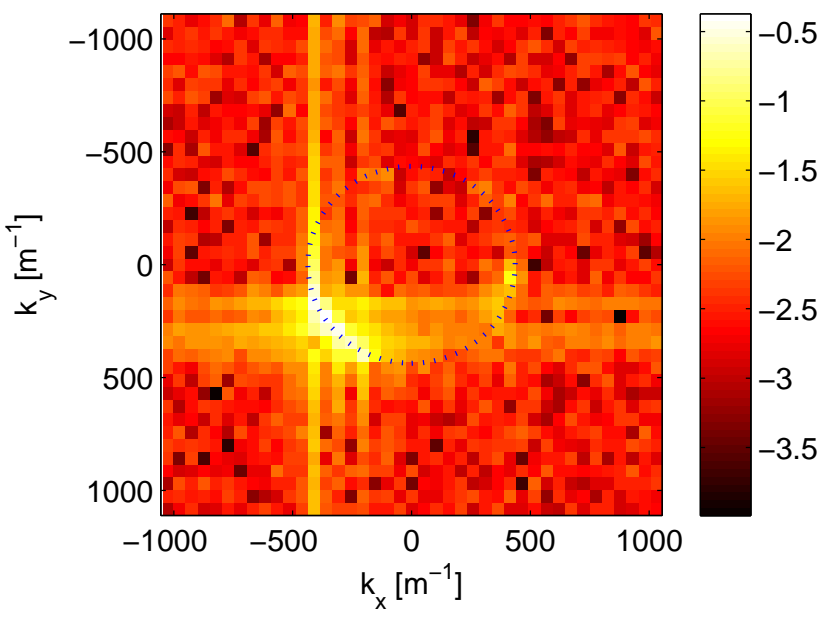

Figure 15: (color online) Spatial Fourier transform amplitude (logarithmic scale) of the measurement data, frequency $15 \mathrm{kHz}$. The dotted circle indicates the estimate of the real part of the wavenumber at this frequency, using the wavenumber fitting approach.

significant pair-wise correlation between the fitting parameters, making a most squares analysis [18,19] unnecessary in this specific case.

Using the wavenumber fitting approach based upon the Hankel's functions and image source method, the obtained wavenumber domain resolution (of the real part of $k$ ) outperforms the wavenumber domain resolution that can be obtained by means of a classical 2D spatial Fourier transform. Figure 15 shows the wavenumber domain spectrum for a frequency of $15 \mathrm{kHz}$, as an example. The typical wavenumber domain resolution of the spatial Fourier transform equals $d k=2 \pi / L$ [14], where $L$ is a typical dimension of the measurement area in either the $x$ or the $y$-direction. In this specific case with a measurement area of 11.7 $\mathrm{x} 11.1 \mathrm{~cm}^{2}$, the wavenumber resolution of the spatial Fourier transform equals $51 \mathrm{~m}^{-1}$ and $57 \mathrm{~m}^{-1}$ in $x$ and $y$-direction, respectively. The uncertainty, and thus the resolution of the real part of the wavenumber using the Hankel-function based wavenumber fitting approach is better than $1 \mathrm{~m}^{-1}$, and a relative uncertainty of less than $2 \%$, as can be seen from Fig. 14 . This is an improvement of the wavenumber resolution by a factor 50. Giving the relationship in Eq. (14), this relative uncertainty of about $2 \%$ in the estimate of $k$ when using the wavenumber fitting approach, gives a relative uncertainty in the estimate of Young's modulus

Submitted to Journal of Sound and Vibration. Accepted 5 December 2016 http://dx.doi.org/10.1016/j.jsv.2016.11.037 
which is in the order of $4 \times 2 \%=8 \%$. The uncertainty due to the influence of the image source model (as discussed in Section 3 ) is much less (for the Young's modulus it was estimated at only $2 \%$ ).

The imaginary part of the wavenumber (or, equivalently, the loss factor $\eta$ of the plate) is more difficult to estimate, because of the fact that the imaginary wavenumbers are much smaller in magnitude as compared to the real part of $k$. This makes that the relative error is much bigger. Nevertheless, the loss factor $\eta$ of the plate can be estimated reasonably well, up to very high frequencies (see Fig. $11 \mathrm{~d})$.

\section{Conclusions}

A new wave fitting approach was presented to estimate the frequency dependent material properties of thin plate structures from an experimentally obtained vibrational field of a point excited plate. An image source model was successfully applied, using Hankel's functions, to describe the vibrational field of a point excited plate with free boundary conditions. Bayesian regularization is used to determine the strengths of the image sources. The (complex valued) wavenumber is varied to minimize the error between the measured and predicted vibrational field. Using this wave fitting procedure, the complex wavenumber is determined as function of frequency, from which the material properties of the plate can be extracted. The complex wavenumber fitting procedure outperforms the classical spatial Fourier transform approach in terms of the wavenumber resolution by a factor 50 , in this experiment.

The method is applied to a sandwich plate. From the estimated wavenumber, the Young's modulus of an "equivalent" Kirchhoff plate was estimated. This approach can be helpful for the modeling of composite plates in the high frequency range, without the need to use onerous $3 \mathrm{D}$ elements or composites theory. Instead, a relatively simple (Kirchhoff) 2D plate model with a frequency dependent "equivalent Young's modulus" can be used.

The method is specifically suited for the higher frequency range, where the wavelength is sufficiently small as compared to the dimension of the plate, and where the damping is sufficiently high to ensure that the vibration field can be explained by a limited number of image sources. Whilst a sufficient number of image sources is required to project the measured data on to the image source model with sufficient accuracy, it was found that the estimation of the (complex) wavenumber and thus the estimation of the Young's modulus and loss factor is not

Submitted to Journal of Sound and Vibration. Accepted 5 December 2016

http://dx.doi.org/10.1016/j.jsv.2016.11.037 
significantly affected by the chosen number of image sources.

\section{Acknowledgments}

This work was supported by the Labex CeLyA of Université de Lyon, operated by the French National Research Agency (ANR-10-LABX-0060/ ANR-11IDEX-0007) and by INSA-Lyon (BQR VIVARIUM project). Prof. Christ Glorieux of KULeuven is acknowledged for his suggestions during the writing of the manuscript.

\section{A Bayesian regularization}

A bayesian formalism [13] is used to find a solution $\alpha$ to the linear system

$$
w=\Phi \alpha+\beta
$$

where $w$ is the observation, $\Phi$ the basis on which $w$ has to be expanded, $\alpha$ the unknown projection coefficients and $\beta$ the unknown projection error. Let's consider the following a priori probability density functions (pdfs), where $\mathcal{N}_{c}(\mu, \mathbf{C})$ stands for a multivariate complex normal distribution with an expected value $\mu$ and covariance matrix $\mathbf{C}$.

$$
\begin{aligned}
& {[\beta]=\mathcal{N}_{c}\left(\mathbf{0}, b^{2} \mathbf{I}\right)} \\
& {[\alpha]=\mathcal{N}_{c}\left(\mathbf{0}, a^{2} \mathbf{I}\right)}
\end{aligned}
$$

The first pdf $[\beta]$ assumes that the noise is gaussian, centred, and of same energy on all dimensions. The second one $[\alpha]$ assumes that the unknown $\alpha$ also follows a centred gaussian law. This a priori assumption puts a higher probability to values of $\alpha$ close to zero, whilst larger values of $\alpha$ are a priori assumed to be less probable. A third pdf is formulated for $w$ considering the two former ones and Eq. (19):

$$
[w \mid \alpha]=\mathcal{N}_{c}\left(\Phi \alpha, b^{2} \mathbf{I}\right)
$$

The Bayes rule allows to invert this conditional pdf as follows

$$
[\alpha \mid w] \propto[w \mid \alpha][\alpha]
$$

An estimate of the unknown $\alpha$ can be obtained by maximizing this posterior pdf, leading to the standard Tikhonov solution:

$$
\hat{\alpha}=\operatorname{argmin}\left(\|w-\Phi \alpha\|^{2}+\lambda^{2}\|\alpha\|^{2}\right)=\left(\Phi^{*} \Phi+\lambda^{2} \mathbf{I}\right)^{-1} \Phi^{*} w
$$

Submitted to Journal of Sound and Vibration. Accepted 5 December 2016 http://dx.doi.org/10.1016/j.jsv.2016.11.037 
where $\lambda^{2}=b^{2} / a^{2}$ and where ${ }^{*}$ denotes the complex conjugate transpose. The benefits of the bayesian frameworks is that the meta parameters $\left(a^{2}, \lambda^{2}\right)$ can also be considered as random variables and inferred from the measurement data. Assuming no a priori information on $\left(a^{2}, \lambda^{2}\right)$, i.e. $\left[a^{2}\right]=\left[\lambda^{2}\right] \propto 1$, the Bayes rule gives

$$
\left[a^{2}, \lambda^{2} \mid w\right] \propto\left[w \mid a^{2}, \lambda^{2}\right]=\mathcal{N}_{c}\left(0, a^{2}\left(\Phi \Phi^{*}+\lambda^{2} \mathbf{I}\right)\right)
$$

The value of $\lambda^{2}$ maximizing this probability density function is expressed through a singular value decomposition of $\Phi=\sum_{k=1}^{N} s_{k} \mathbf{u}_{\mathbf{k}} \mathbf{v}_{\mathbf{k}}{ }^{\prime}$ (with $N$ the dimension of $\alpha)$ :

$$
\hat{\lambda^{2}}=\operatorname{argmin}\left(\sum_{k=1}^{N} \ln \left(s_{k}^{2}+\lambda^{2}\right)+N \ln \left(\sum_{k=1}^{N} \frac{\left|\mathbf{u}_{\mathbf{k}}{ }^{\prime} w\right|^{2}}{s_{k}^{2}+\lambda^{2}}\right)\right)
$$

The minimization is realized numerically in this work. A set of candidate values of $\lambda^{2}$ is sampled between bounds defined as a function of the minimum and maximum values of squared singular values $s_{k}^{2}$, and the value minimizing this function is selected.

\section{References}

[1] J. G. McDaniel, P. Dupont, L. Salvino, A wave approach to estimating frequency-dependent damping under transient loading, Journal of Sound and Vibration 231 (2) (2000) 433-449, 25. URL <GotoISI> : / /WOS: 000086289200009

[2] J. Berthaut, M. N. Ichchou, L. Jezequel, K-space identification of apparent structural behaviour, Journal of Sound and Vibration 280 (3-5) (2005) 11251131, iCHCHOU, Mohamed/M-5352-2013 ICHCHOU, Mohamed/00000002-2452-0831 13.

URL <GotoISI> : / /WOS : 000226699900036

[3] R. Cherif, J.-D. Chazot, N. Atalla, Damping loss factor estimation of twodimensional orthotropic structures from a displacement field measurement, Journal of Sound and Vibration 356 (2015) 61-71, 0.

URL : / /WOS: 000359316600005

[4] K. Grosh, E. G. Williams, Complex wave-number decomposition of structural vibrations, Journal of the Acoustical Society of America 93 (2) (1993)

Submitted to Journal of Sound and Vibration. Accepted 5 December 2016 http://dx.doi.org/10.1016/j.jsv.2016.11.037 
836-848, grosh, Karl/0000-0001-8704-6199 22.

URL : / /WOS:A1993KL82000023

[5] J. Cuenca, F. Gautier, L. Simon, The image source method for calculating the vibrations of simply supported convex polygonal plates, Journal of Sound and Vibration 322 (4-5) (2009) 1048-1069, cuenca, Jacques/00000003-4503-4151 11.

URL <GotoISI>: / /WOS: 000265793300028

[6] R. Gunda, S. M. Vijayakar, R. Singh, Method of images for the harmonic response of beams and rectangular-plates, Journal of Sound and Vibration 185 (5) (1995) 791-808, 16.

URL <GotoISI> : / /WOS :A1995RU18000003

[7] F. G. J. Cuenca, L. Simon., Measurement of complex bending stiffness of a flat panel covered with a viscoelastic layer using the image source method., in: Euronoise 2009, Edinburgh, European Acoustics Association (EAA), 2009.

[8] R. Gunda, S. M. Vijayakar, R. Singh, J. E. Farstad, Harmonic Green's functions of a semi-infinite plate with clamped or free edges, Journal of the Acoustical Society of America 103 (2) (1998)

[9] A. W. Leissa, Vibration of Plates (Acoustical Society of America, Woodbury, NY, 1993), 2nd ed.

[10] J. Cuenca, F. Gautier, L. Simon, Harmonic green's functions for flexural waves in semi-infinite plates with arbitrary boundary conditions and highfrequency approximation for convex polygonal plates, Journal of Sound and Vibration 331 (6) (2012) 1426-1440, cuenca, Jacques/0000-0003-450341514.

URL <GotoISI> : / /WOS : 000299447700014

[11] S. H. Yoon, P. A. Nelson, Estimation of acoustic source strength by inverse methods: Part ii, experimental investigation of methods for choosing regularization parameters, Journal of Sound and Vibration 233 (4) (2000) 669-705. URL : / /WOS:000087679000007

[12] J. Antoni, A bayesian approach to sound source reconstruction: Optimal basis, regularization, and focusing, The Journal of the Acoustical Society of

Submitted to Journal of Sound and Vibration. Accepted 5 December 2016

http://dx.doi.org/10.1016/j.jsv.2016.11.037 
America 131 (4) (2012) 2873-2890.

URL http://scitation.aip.org/content/asa/journal/ jasa/131/4/10.1121/1.3685484

[13] A. Pereira, J. Antoni, Q. Leclere, Empirical bayesian regularization of the inverse acoustic problem, Applied Acoustics 97 (2015) 11-29, 0.

URL : / /WOS : 000355495900002

[14] E. G. Williams, Fourier Acoustics; Sound Radiation and Nearfield Acoustical Holography, Elsevier, New York, 1999.

[15] M. Rak, M. Ichchou, J. Holnicki-Szulc, Identification of structural loss factor from spatially distributed measurements on beams with viscoelastic layer, Journal of Sound and Vibration 310 (4-5) (2008) 801-811, holnickiSzulc, Jan/L-2985-2013; ICHCHOU, Mohamed/M-5352-2013 HolnickiSzulc, Jan/0000-0001-9982-3979; ICHCHOU, Mohamed/0000-0002-245208319.

URL <GotoISI> : / /WOS: 000253646000005

[16] P. M. Morse, K. U. Ingard, Theoretical Acoustics, Princeton University Press, 1987.

[17] P. R. Bevington, D. K. Robinson, Data Reduction and Error Analysis for the Physical Sciences, McGrawHill, New York, 2003.

[18] J. Sermeus, B. Verstraeten, R. Salenbien, P. Pobedinskas, K. Haenen, C. Glorieux, Determination of elastic and thermal properties of a thin nanocrystalline diamond coating using all-optical methods, Thin Solid Films 590 (2015) 284-292.

URL : / /WOS: 000361057100043

[19] D. D. Jackson, Most squares inversion, Journal of Geophysical Research 81 (5) (1976) 1027-1030.

URL : / /WOS:A1976BG75400032

Submitted to Journal of Sound and Vibration. Accepted 5 December 2016

http://dx.doi.org/10.1016/j.jsv.2016.11.037 